\title{
An End-to-end Early-warning System based on Wireless Sensor Network for Gas Leakage Detection in Industrial Facilities
}

\author{
Haythem Bany Salameh, Senior Member, IEEE, Mohammad Dhainat and Elhadj Benkhelifa
}

\begin{abstract}
Existing Liquefied Petroleum Gas (LPG)-detection systems are ad-hoc and designed as stand-alone nodes. This paper, however, presents an integrated end-to-end Wireless Sensor Network (WSN) system that integrates hardware and software for early-warning gas-leakage detection and monitoring applications; fully utilizing the Internet-of-thing (IoT) functionalities and capabilities in WSNs at the network level such that network performance is improved. The proposed system can operate in single-hop and multi-hop modes depending on the surrounding radio frequency (RF) environment and network topology. Specifically, we design a per-deployed WSN system for LPGgas detection/monitoring in residential areas and factories that collects, analyzes and forwards the collected information over a wireless channel to the monitoring center to take the appropriate action. To achieve a reliable communication and data delivery, we implement an efficient communication protocol that organizes the data exchanges between the different nodes in the network. The proposed WSN system is deployed and experimentally tested. The data acquired from the various experiments is used to examine the reliable operation of the implemented system in terms of robustness and data-delivery reliability. Robust and reliable performance is demonstrated with packet loss rate as low as $5 \%$. The experimental results also indicate that the proposed system can promptly detect gas-leakage within $50 \mathrm{~ms}$ and provide accurate gas concentration measurements with $97 \%$ accuracy.
\end{abstract}

Keywords-WSN, IoT, Gas Leakage Detection, End-to-End System, Remote Sensing.

\section{INTRODUCTION}

Wireless Sensor Networks (WSNs) is considered an essential enabler for effective IoT services and applications [1][8]. WSNs are widely used in several civilian and military applications including environmental surveillance, monitoring,

Haythem Bany Salameh is with the Department of Network and Communications Engineering, Al Ain University, Al Ain, United Arab Emirates, with the Department of Telecommunications Engineering, Yarmouk University, Irbid 21163, Jordan, and also with Cloud Computing and Applications Research Lab, Staffordshire University, UK (e-mail: haythem.banysalameh@aaue.ae.ac).

Mohammad Dhainat is with the Department of Telecommunications Engineering, Yarmouk University, Irbid 21163, Jordan

Elhadj Benkhelifa is with Cloud Computing and Applications Research Lab, Staffordshire University, Stoke-on-Trent ST4 2DEUK, and also with University of Suffolk, UK.

This research was supported in parts by the QR Global Challenges Research Fund (GCRF), Staffordshire University, UK and the Science Research Support Foundation (SRF)-Amman-Jordan. A limited subset of initial results was preliminarily presented at the IEEE SDS'2016 Conference, AZ, USA. health-care services, data collection, etc. [8]-[10]. WSNs can be easily deployed without the need of any pre-existing infrastructure and can be used for unattended operation with limited maintenance. Each sensor node has a sensing device that can measure and report the observed measurements of some physical conditions (e.g. detect Liquefied Petroleum Gas (LPG) leakage $\left.{ }^{1}[12]-[16]\right)$. The sensed data may be processed and aggregated before being communicated to the command center [11].

Liquefied Petroleum Gas (LPG) is one of our daily life necessities, in almost all residential locations and service areas (i.e., houses, factories, restaurants, hotels). However, any undetected leakage of gas may cause serious fires, business loss and fatalities. Thus, it is important for any leakage to be detected and take the appropriate action in a timely manner. For this purpose, a WSN can be pre-deployed to remotely monitor possible gas leakage, in order to control its spread and avoid potential catastrophes. On the other hand, fire-fighting is one of the most dangerous, yet important professions. The dangers associated with this profession are contributed to the limited information related to the location, distribution, size and spread of the fire, when it is caused by gas leakage.

Several theoretical and practical attempts have been made to use WSN in gas-leakage detection and firefighting applications [15]- [23], but they still suffer from a number of drawbacks. A full survey on related work and existing systems was published by the authors in [24], for further details. The main out outcome of this published survey paper is that existing practical LPG-detection systems are ad-hoc and designed as stand-alone nodes, where as the theoretical proposed WSN systems are not experimentally tested (not practically implemented). In addition, most of the the theoretical efforts attempted to solve a sub-set of the design problem such as providing communication protocols for WSNs, clustering mechanism, routing, etc. This paper, however, presents an integrated end-to-end WSN system that integrates hardware and software for earlywarning gas-leakage detection and monitoring applications. Unlike existing systems, our proposed solution, include new communication protocols and hardware components that fully utilize the IoT functionalities and capabilities in WSNs at the network level such that network performance is improved. Hence, to practically realise this, we develop a customized end-to-end system based on inexpensive locally manufac-

\footnotetext{
${ }^{1}$ Gas leak detection is the process of identifying potentially hazardous gas leaks using several sensing devices.
} 
tured wireless sensor devices that includes hardware design, supporting software, testing, fabrication and implementation. The proposed system features a reliable sensing and data processing algorithms as well as efficient distributed communication mechanisms. The adopted communication protocol provides reliable data delivery while being energy-efficient. The proposed system collects, analyzes and transmits the sensed data over a wireless channel to a monitoring center over the Internet to take the appropriate action/decision. To verify the effective operation of the developed end-to-end WSN system, we perform realistic testing experiments over a testbed of 15 nodes pre-deployed WSN. The collected data and gas-concentration measurements reveal that the proposed endto-end early-warning system can remotely provide accurate, reliable and timely alerts. Such alerts include accurate reported gas concentration levels along with the associated locations.

The rest of the paper is organized as follows. In Section II, we provide an overview of WSN technology. The different WSN design variants and requirements for LPG monitoring/fire-fighting applications are discussed in Section II-B. Section III illustrates the hardware and tools used in developing our end-to-end system. The hardware and software design and implementation of the end-to-end system are discussed in Section IV. The testbed and experimental results are given in Section VI. In Section VII, we conclude this paper.

\section{PRELIMINARIES}

\section{A. WSN Overview}

A WSN is a wireless network of a number of batterypowered self-organized sensor devices that are responsible for collecting information regarding some environment phenomenon and conditions. To be energy-efficient, the sensor nodes perform signal processing to the raw data before forwarding such data to a central node (sink node), which can be connected to the Internet to form an IoT-based WSN [25]. In such systems, information technology, software, hardware, networking and programming aspects are integrated. Specifically, each sensor node often contains three key inter-connected subsystems: (1) radio transceivers, (2) sensing devices and (3) processor with memory device.

\section{B. WSN Solutions for Gas leakage Monitoring and Fire- fighting Applications}

Acquiring data from inside of a closed area in fire is a challenging problem for firefighters. In these cases, data is vital to facilitate the firefighters' operations, minimize damages and possibly save lives. WSNs can be deployed for this purpose [24] to remotely acquire the following types of data:

- The proximity of fire-fighters to danger, obtained using temperature, smoke, oxygen, and olfactory sensors.

- The likelihood of flashover, obtained using temperature sensor.

- The possibility of Backdrafts, obtained using oxygen sensor.

- Hidden fires, obtained using temperature and smoke sensors.
- Structural collapse issues, obtained using Accelerometers sensor.

- Gas concentration (leakage), obtained using gas sensor. Depending on the application requirements, each sensor node in the deployed WSN can be equipped with one (or more) kind of the aforementioned sensitive sensor units. In this paper, we consider the design of an end-to-end pre-deployed WSN system, where each user is equipped with a LPG sensor module. Specifically, we focus on the various challenges that must be addressed for the deployment and use of a WSN for early-detection of gas-leakage and subsequent spread of gas in closed areas and building. The proposed system is a clusterbased WSN that is suitable for gas monitoring applications. Using clustering facilitates scalable network operations. In our developed cluster-based WSN, each sensor node in a given cluster can only create connectivity with its cluster-head node, whereas each cluster-head node can create a mesh connectivity with any other cluster-head node in the network such that the data can be delivered to the sink node.

\section{The Proposed End-To-End SyStem}

\section{A. System Overview}

The proposed end-to-end system is based on inexpensive locally manufactured (designed and implemented) wireless sensor devices. The proposed system is a cluster-based WSN that is suitable for gas monitoring applications. Using clustering facilitates scalable and energy-efficient network operations. In our system, each sensor node in a given cluster can only create connectivity with its cluster-head node, whereas each cluster-head node can create a mesh connectivity with any other cluster-head node in the network such that the sensed data can be delivered to the sink node and then over the Internet to the monitoring center to take the appropriate action/decision. Hence, the proposed design can operate in single-hop and multi-hop modes depending on the surrounding RF environment and network topology [27]. Figure 1 shows an example of our developed pre-deployed cluster-based WSN system with three clusters. In the next sub-section, we describe the main hardware components and software tools that are used in developing and testing our end-to-end system.

\section{B. System Components}

1) RF Transceiver Module: The communication and data transfer between the sensor nodes is based on the IEEE802.15.4 standard. We consider the ZigBee embedded RF modules (Series 1 XBee 4214A and XBee pro) with free protocol stack. These modules provide simple and very reliable communications between the various sensor nodes (i.e. pointto-point and point-to multi networks). Zigbee Xbee series 1 module provides cost-effective wireless connectivity in the unlicensed ISM band with built-in antennas and supports interoperable operation with other ZigBee devices. Specifically, XBee modules that enable software-intelligent networking using the XBee ZigBee X-CTU software. The XBee modules can be reprogrammed such that customized transmission functionalities can be realized. The XBee modules support a maximum 


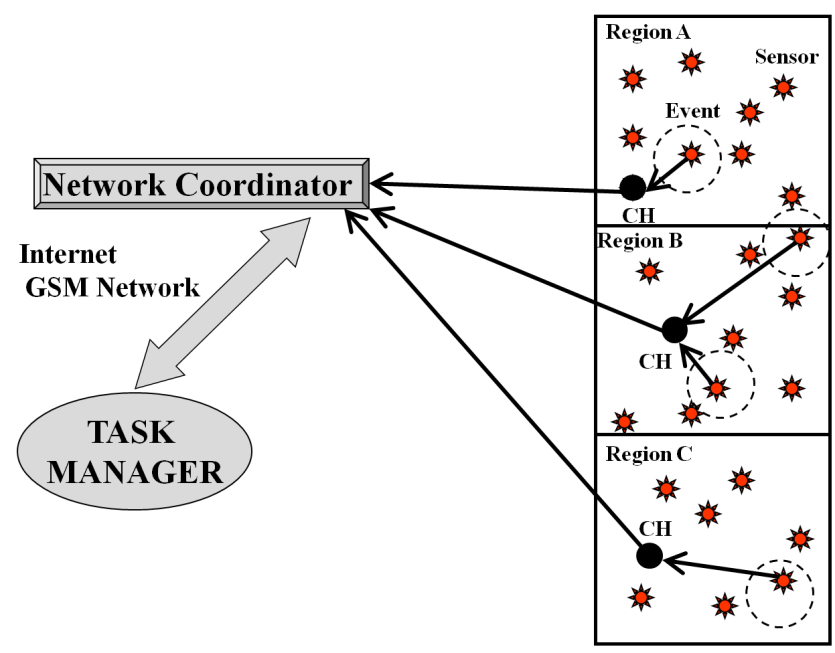

Fig. 1. A pre-deployed GSM-enabled end-to-end WSN with three regions.

data rate of $250 \mathrm{Kbps}$ at $2.4 \mathrm{GHz}$ operating frequency. The maximum transmission (output) power of the XBee and XBeePRO modules are respectively 1 and $10 \mathrm{mw}$, with receiver sensitivity of $-92 \mathrm{dBm}$ and $-100 \mathrm{dBm}$, respectively. Thus, the transmission ranges for the XBee and XBee-PRO are 90 and 750 meter, respectively [30]. In our design, we assume XBee module for the sensor node in each cluster (to achieve indoor/Urban range) and XBee-PRO module for cluster-head nodes (to achieve outdoor range).

2) Gas Sensor Module: A number of gas sensor devices exist with different specifications based on the needed sensitivity, type of sensed gas, and the size. For our purposes, we consider the MQ-6 gas sensor, which has a tunable resistor $\left(R_{t}\right)$ on board, for adjusting the sensor sensitivity and identifying the gas to be sensed. The MQ-6 is a small-size low power consumption sensor with extremely high sensitivity and very rapid response rate. This sensor is sensitive to LPG, propane and iso-butane. This makes it suitable to be considered in gasleakage detection applications. The tunable resistor $R_{t}$ can be used to increase the sensing sensitivity of a specific types of gas (e.g., for butane gas detection, $R_{t}=20 \mathrm{~K} \Omega$ ). The MQ6 sensor can sense the concentrations of flammable butane gas and provides the concentration level in a form of analog voltage. According to the data-sheet of the sensing device, each voltage represents a given level of concentration. We use the data-sheet to program the micro-controller such that the reported voltage is represented in a form of the required measurement. Hence, the sensor calibration can be done with the help of the data-sheet. Specifically, the sensor can measure LPG levels with concentration ranges from 200 to $10000 \mathrm{ppm}$ by generate a voltage ranges from 0.1 to $5 \mathrm{~V}$. The sensor operates with $150 \mathrm{~mA}$ at $5 \mathrm{~V}$. It also provides stable operation at temperatures ranging from -10 to $50{ }^{\circ} \mathrm{C}$. In summary we have selected the MQ-6 sensor because of its following features: (i) wide detecting range, (ii) high sensitivity, (iii) fast response, (iv) long expected lifetime, (v) operation stability, (vi) simplifies drive circuit and (vii) compact size with low cost.

3) Micro-controller/Processor Module: We consider the PIC18 (XPic Micro-controller 18F4620) as the processing unit in the developed sensor nodes. This processor is considered because of its small-size and low cost. In addition, this microcontroller can be easily configured to control several devices based on sensing input from the real world. XPic microcontroller contains built-in A/D converter and a relatively sufficient memory size of $1 \mathrm{MB}$, which allows multiple function to be performed. The PIC18 module supports a number of attractive features including: a 21 bits read-and-write capable call stack, conditional branch instructions, indexed addressing, extendable registers up to 12 bits, and additional registers up to 3 .

4) Power Supply: We consider rechargeable Lithium-ion batteries as a power supply in the developed sensor nodes. Lithium-ion batteries have been selected as they have high charge density (long life). Depending on the chemical compounds and used design, voltages from $1.5 \mathrm{~V}$ to $3.7 \mathrm{~V}$ can be produced by lithium cells. Based on the role of each node in our design, some of the nodes will be equipped with micoUSB socket such that the nodes are directly powered from the AC main-lines using AC/DC adapters. In this case, the battery is used as a backup power unit. Other nodes are batterypowered. In such nodes, power-level indicator circuit will be implemented to alert the network administrator.

Battery voltage-level indicator: In our design, we implement a simple battery-voltage indicator circuit using a tricolor led. The tricolor led is connected to the PIC micro-controller, which is responsible of reading the battery voltage level. If the voltage level is within $[75-100] \%$ range, the tricolor led will be activated with green color. If the voltage level is within the $[50-75) \%$ range, the tricolor led will be activated with yellow color. If the voltage level is below $50 \%$, the tricolor led will be activated with red color.

Step-up DC-to-DC converter: Because most of the rechargeable batteries provide up to 3.7 volts and our circuit requires 5 volts, we design a step-up DC-to-DC converter using the switch-mode power-supply technology. The circuit design of the step-up/boost converter includes: inductor, capacitor, diode, switch and error amplification element.

5) GSM Module: We consider the Quad-band embedded GSM Module with AT command set (GSM 850/900 1800/1900) and SIM card socket. In addition, an RS232 interface on CMOS level is used with the GSM module. This module can support both TCP/IP stack, UDP/IP stack, embedded SMTP and FTP, which makes it suitable for remote data delivery over the Internet using the cellular network. It has a very small-size of $22 \times 22 \times 3 \mathrm{~mm}$ with relatively lightweight of $3.2 \mathrm{~g}$.

\section{The Design AND IMPLEMENTATION OF THE PROPOSED PRE-DEPLOYED WSN}

In this section, we describe in details the hardware (circuit design) and software (communication and processing protocols) implementations of the developed WSN system. The supporting software components manages the operation of 
the various hardware components and nodes in the system including data-collection, data-processing, channel-access and communication algorithms. This software and hardware integration is done by including a PIC micro-controller in each sensor device, in which the software components (including the programs and codes) are implemented via the PIC microcontroller.

\section{A. Design Overview}

The proposed system is designed for unattended operation, where the LPG concentrations in several closed areas in a given facility can be remotely monitored by pre-deploying the sensor nodes in an appropriate manner. Hence, we use attributebased addressing (i.e., location-based). For such type of data delivery, the data traffic flow is predominantly unidirectional to the command center, in which the traffic is intermittent that demands low transmission speeds. The proposed pre-deployed end-to-end WSN system is considered as an energy-efficient event-driven system, in which the collected information is only transmitted to the monitoring center over the Internet when the LPG level is above a dangerous threshold level. In particular, the sensor nodes remain into a sleep mode if there is no event to report, which preserves their precious limited energy and extend the network lifetime.

In our system, we define four different types of nodes: a sensor node, a cluster-head node, a wireless coordinator node, and a sink (i.e., task manager) node (see Figure 1). According to our system design, the area to be monitored is sub-divided into a set of sub-areas (clusters). Each cluster includes a cluster-head and a group of sensor nodes. A predefined configuration is used to manually deploy the sensor nodes in each cluster to achieve the best performance. Each sensor node includes a short-range ZigBee transceiver (i.e., 90 meter) [28], [29] (inline with the IEEE 802.15.4 standard). Cluster-heads are equipped with ZigBee transceivers that form a network of cluster-heads. The key task of each sensor node in a given cluster is to sense the LPG measurements in that cluster using the MQ-6 sensing device and communicate the measured data to its cluster-head in case of gas-leakage (event) detection. Then, the cluster-heads, wirelessly, transfer the alarm packets to the sink node (i.e. the network coordinator), which contains the gas concentration and the location of gas leakage. The cluster heads forward their data packets to the coordinator. The cluster-heads perform data collection, compression and delivery to the sink node. A centralized mechanism is used to perform channel assignment while keeping in mind the importance of the collected alarms from the cluster heads (the LPG level). The coordinator transmits the received alarm packets to the task manager (e.g., fire-fighting department) through the Internet to take the appropriate action. Specifically, the Internet layer with its services is implemented at the network coordinator to deliver the reported LPG leakage information over the Internet to the task manager. The network coordinator can also control the gas-leakage source by the wireless actuator when available or can trigger a buzzer to alert people of the gas hazard. In summery, the sensor nodes in each cluster perform accurate LPG leakage detection, whereas the cluster-heads are responsible of delivering the detected events to the network coordinator and delivering such information over the Internet to the task manager.

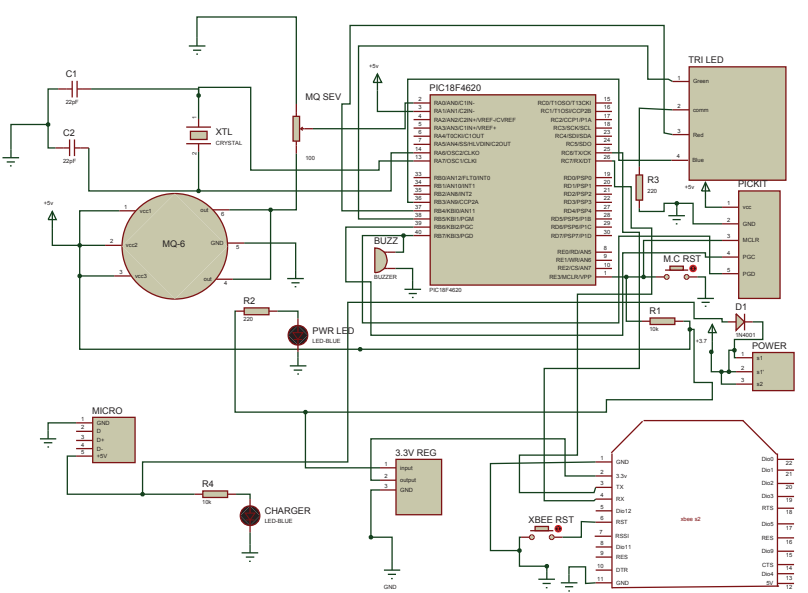

(a) Schematic diagram

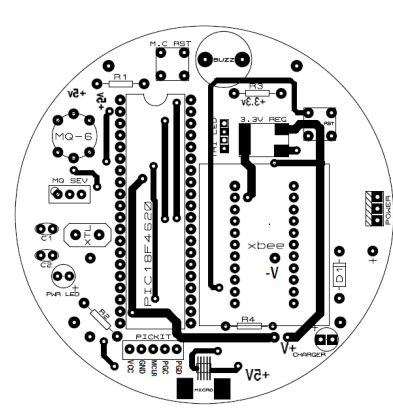

(b) PCB upper-layer design

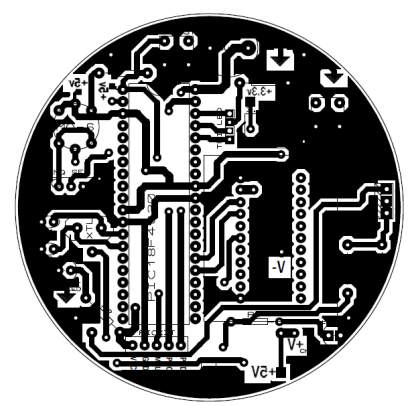

(c) PCB lower-layer design
Fig. 2. Sensor node circuit design.

\section{B. Hardware Implementation}

- Sensor Node: The circuit of a sensor node consists of: (1) MQ6-gas sensing device with tunable resister, (2) XPic micro-controller, (3) short-range XBee transceiver, (4) ion-lithium batteries with micro-usb charger socket, and (5) On/Off switch. Figure 2 (a) shows the sensor node circuit schematic diagram, while Figures 2 (b) and (c) present the lower-layer and upper-layer PCB design.

- Cluster-head Node: The circuit of a cluster-head consists of: (1) XPic micro-controller, (2) long-range XBeePRO transceiver, (3) ion-lithium batteries with microusb charger socket, and (4) On/Off switch. Figure 3 (a) shows the cluster-head node circuit schematic diagram, while Figures 3 (b) and (c) present the lower-layer and upper-layer PCB design.

- Sink/Coordinator Node: The design of the sink node consists of: (1) LCD monitor, (2) XPic micro-controller, 
(3) On/Off switch, long-range XBee-PRO transceiver, (4) ion-lithium batteries that has a micro-usb charger socket and (5) a GSM Module. Figure 4 (a) shows the sink node circuit schematic diagram, while Figures 4 (b) and (c) present the lower- and upper-layer PCB design.

- Task-manager Node: The circuit design of the task manager node is a GSM phone.

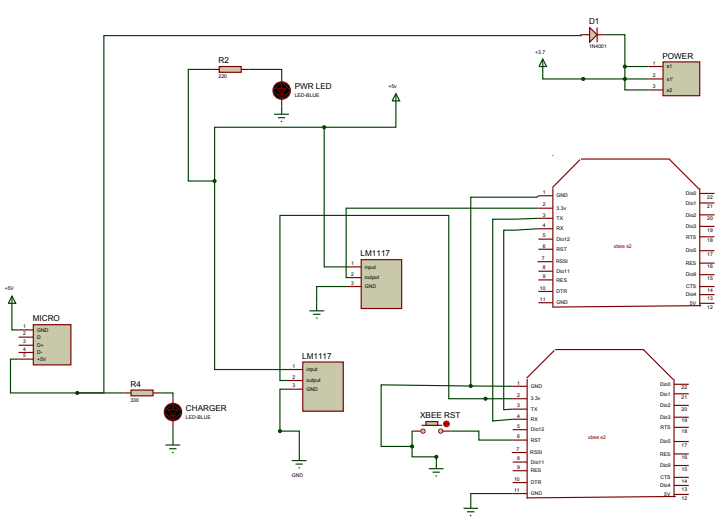

(a) Schematic diagram

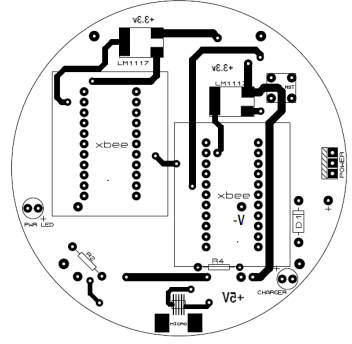

(b) PCB upper-layer design

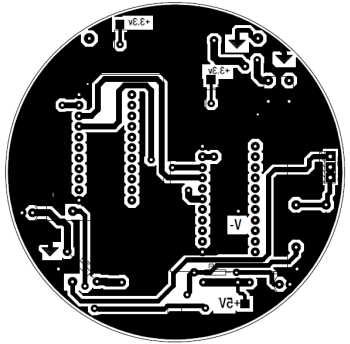

(c) PCB lower-layer design
Fig. 3. Cluster-head circuit design.

\section{Software Implementation and Communication Protocols}

Because of the hierarchical architecture of the designed pre-deployed WSN, we adopt the event-driven sensor MAC (EDS-MAC) protocol, the details of which can be found in a previously published paper, by the Authors in [26]. This protocol has two MAC-layer communication levels: CSMAlike intra-cluster and multi-channel CSMA-based inter-cluster communications. The intra-cluster communications are performed in each cluster, in which the sensor nodes use the CSMA/CA protocol that is being used in the IEEE 802.11 standard with similar interframe spacing and collision avoidance strategies. This protocol deactivates the RTS/CTS control exchanges because the length of each data packet is very small. It is also based on an even-driven transmission strategy to save energy (nodes are in sleep mode if no event to report). The detailed operation of the intra-clu er communication protocols is described in Algorithm 1. Accordingly, each cluster-head $(\mathrm{CH})$ has up-to-date data regarding the sensed events and their locations. Then, each cluster-head with received alarm packets forwards them to the network coordinator using one of the ISM channels according to a CSMA-CA-like protocol (inter-cluster communications). The operation details of the inter-cluster channel access protocol are provided in [26] and detailed in Algorithm 2. The time-line diagram of the operation of the adopted protocol is shown in Figure 5.
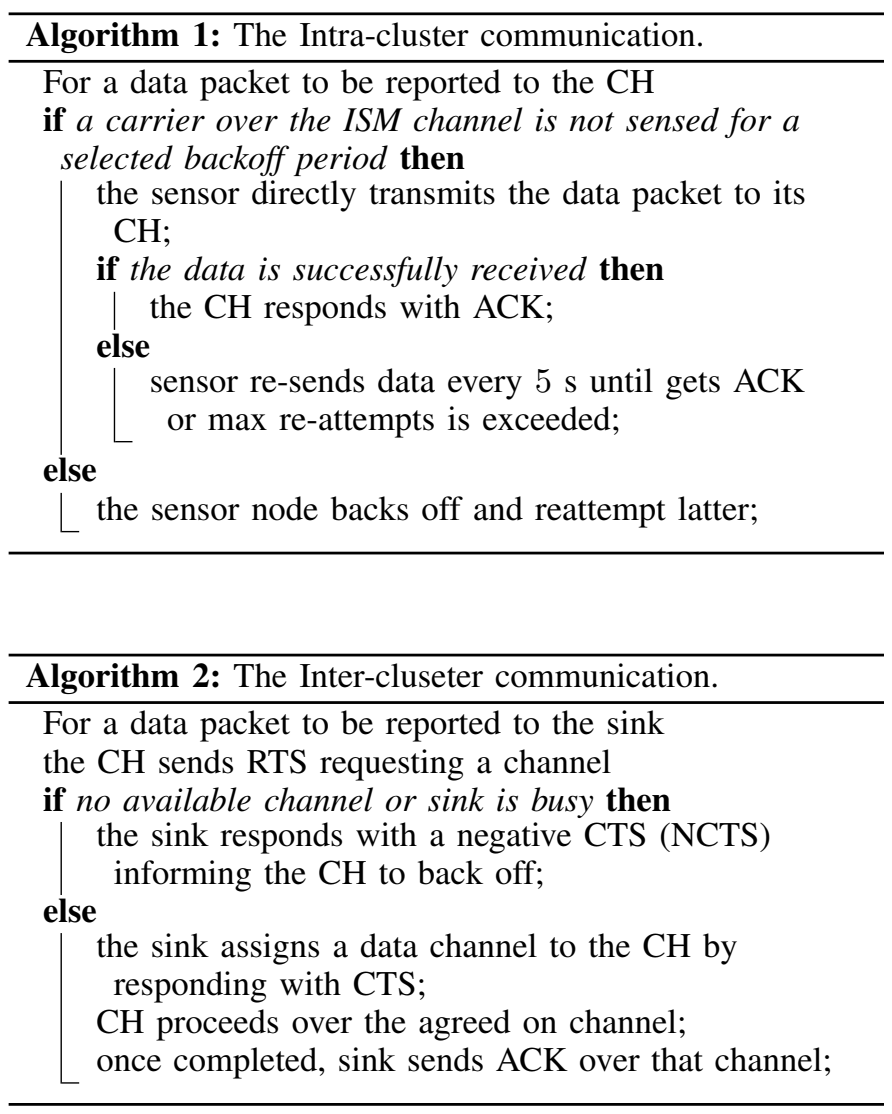

\section{Device Manufacturing: Implementation PROCESS}

The different types of nodes and devices have been manufactured using a two-layer copper PCBs. The PCB manufacturing is performed through the following steps.

\section{A. Design Verification using Software and Breadboards}

The circuit schematics (given in the previous section) contain detailed description of all the used elements and connections. We first test the circuit thoroughly using simulations (proteus 8). Then, we assemble and test each part of the proposed end-to-end system on a breadboard, as it simplifies the verification and testing procedures without the need for solder and/or etches. 


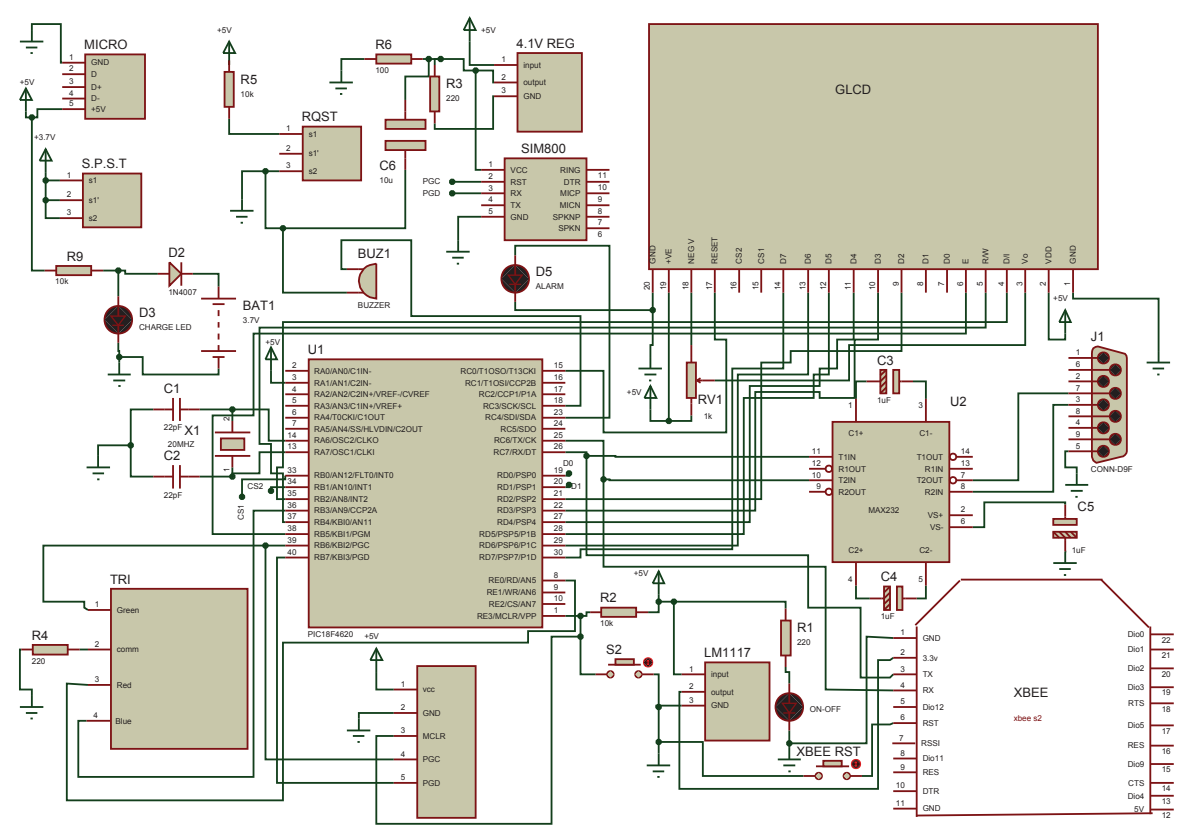

(a) Schematic diagram

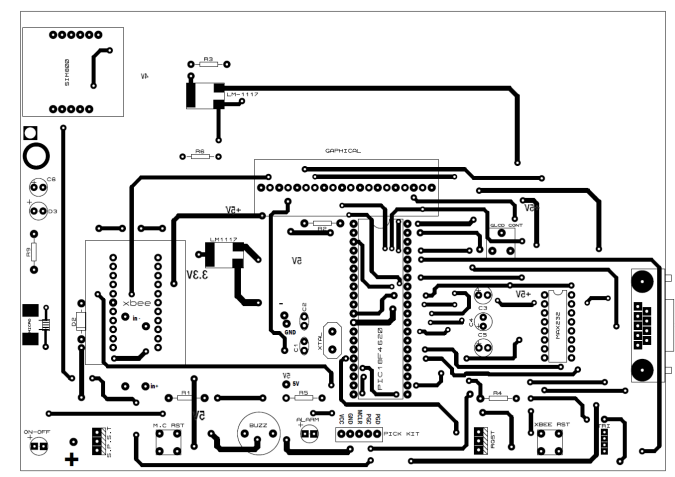

(b) PCB upper-layer design

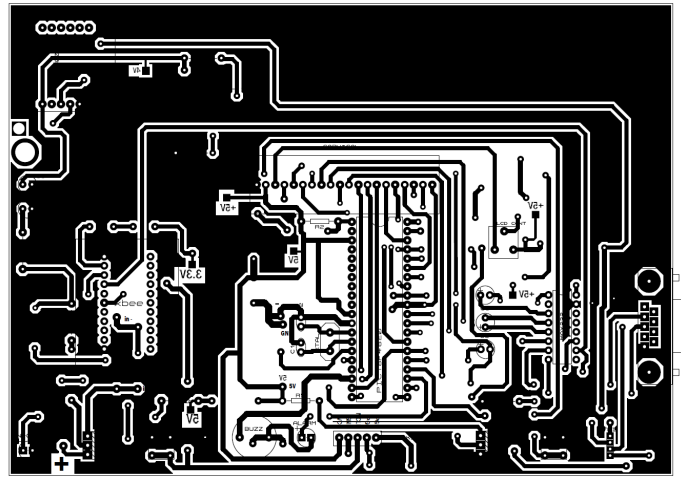

(c) PCB lower-layer design

Fig. 4. Sink node circuit design.

\section{B. PCB Manufacturing Process}

After verifying the circuit functions on the breadboards and the simulation software, we print the circuit schematic on transparency paper. Then, the manufacturing process is conducted as follows:

1) The UV-exposure process: the main purpose of this step is to burn a photo a of the schematic on the raw-material PCB boards.

2) Developing with Chemical etching Process: the main purpose of this step is to remove the un-needed copper material. The etching is done with $\mathrm{NAOH}$ solution.

3) Lamination process: The two-layer printed circuit boards have traced layers inside the board, which has been accomplished using pressure and heat for a limited time, resulting in inseparable one-piece device.

4) Drilling process: The required holes through the PCB boards are drilled using small-diameter drill with solid coated tungsten carbide.

5) Plating and coating process: PCBs are plated with solder over nickel for etching away un-wanted copper. After etching, we apply a coating mask.

6) Bare-board testing process: Unpopulated boards are tested for "shorts" and "opens" circuits.

7) Assembly and soldering Process: PCB with test con- 


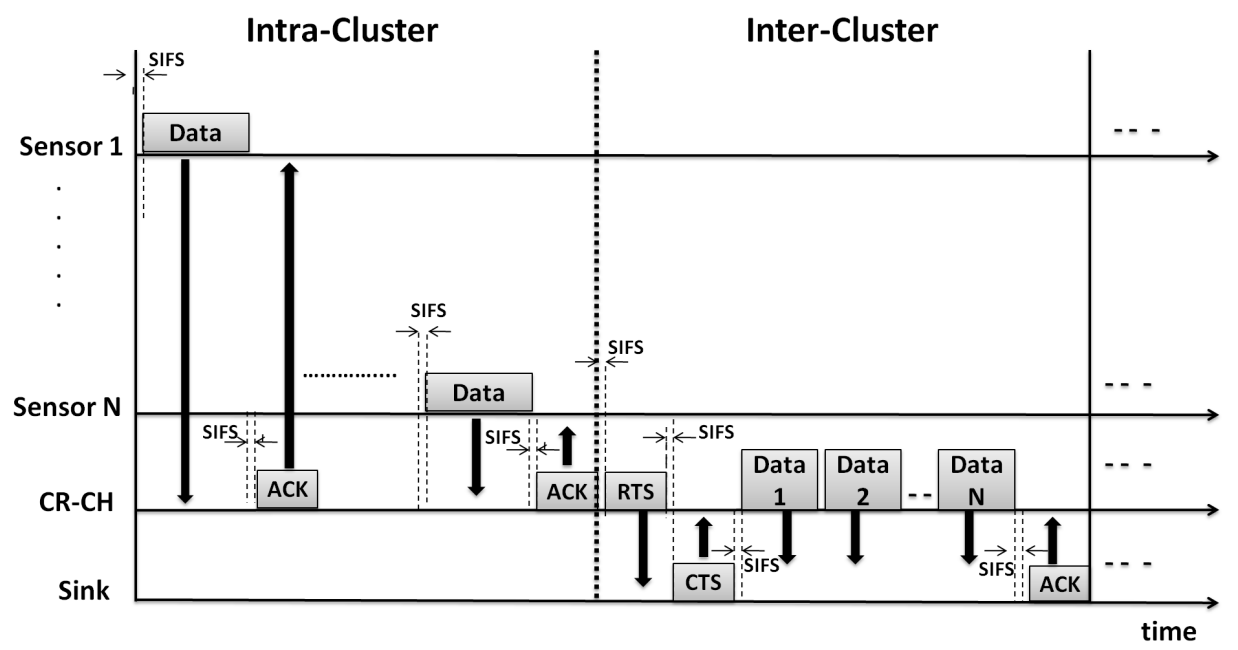

Fig. 5. Basic operation of the adopted MAC Protocol.

nection pads are assembled and populated with the electronic elements according to our design.

8) After populated with elements, each PCB is tested using: power-off testing (PCB component placement and visual inspection) and power-on testing (physical measurements such as voltage and functional test).

9) Protection and packaging process: After finishing the above steps, the PCBs are packaged using thermal hardrubber material (see Figure 6).
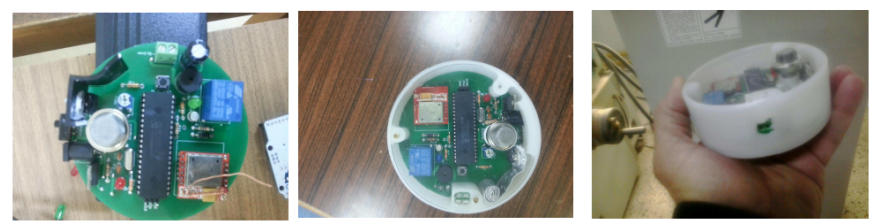

(a) The assembled pre-deployed sensor and cluster-head nodes
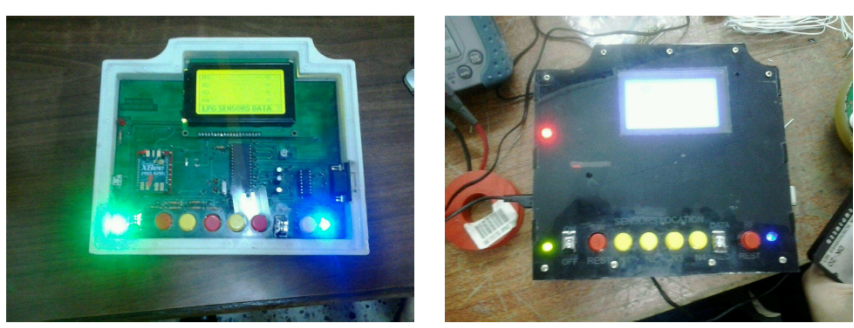

(b) The assembled pre-deployed sink node

Fig. 6. The end-to-end manufactured nodes of the proposed system.

\section{EXPERIMENTAL EVALUATION}

The performance of the proposed end-to-end pre-deployed WSN system is evaluated through testbed experiments to measure the accuracy of the reported LPG level.

\section{A. Experiment Setup}

In our experiments, we implement a cluster-based WSN system by manufacturing three clusters and a sink node, in which each cluster includes a cluster-head and several nodes. Each sensor node in a given cluster can only create connectivity with its cluster-head node, whereas each clusterhead node can send the sensed data to the sink node or create a mesh connectivity with any other cluster-head node in the network such that the data can be delivered to the sink (as depicted in Figure 2). We use an MQ-6 LPG sensing unit at each sensor node to measure the LPG concentration. For intra-cluster communication, we use an XBee-4214A Series 1 ZigBee-embedded RF module in each sensor node operating over the unlicensed 2.4-GHz ISM band. For the intercluster communications between the cluster-heads and the sink, each cluster-head is equipped Two MRF49XA RF modules (operating over the $434 \mathrm{MHz}$ and $868 \mathrm{MHz}$ bands, and one XBee-4214A Series 1 ZigBee module (operating over the 2.4 $\mathrm{GHz}$ band). Such RF devices are demonstrated to provide reliable communications and can perform channel probing to estimate channel quality. These RF modules are reconfigured using the module's firmware (X-CTU software) such that the adopted communication protocols are implemented. We set the transmission power to $10 \mathrm{~mW}$. We note here the module's receiver sensitivity is $-100 \mathrm{dBm}$. Accordingly, the transmission radius of $90 \mathrm{~m}$ (in-door) is achieved [30]. The reported LPG leakage readings are transmitted using a fixed-length data packet of size (24 bits), where 8 bits are authentication and addressing bits, while the remaining 16 bits are used to present the LPG concentration level. This selection gives an accuracy 
of three significant digits (each 8 bits represent a significant figure). Upon successfully receiving a packet, any receiving node respond with an ACK packet of 8 bits.

\section{B. Experiment Results}

We first consider one cluster and measure the LPG concentration for 11 minutes to verify the accuracy, robustness, and communication reliability of the proposed end-to-end WSN system. We note that when no event to report, the sensed LPG level is periodically reported to the sink on a time scale of 10 seconds. Figure 7 shows real-time LPG measurements (measured at the sensor) and the reported levels of LPG (at the sink). It can observed from Figure 7 that our end-to-end WSN system can provide accurate gas-leakage detection and reliable timely gas-concentration reporting. Figure 8 shows the delivered LPG levels at the sink location is shown for two scenarios: no LPG leakage and three LPG leakage instances (each lasts for 15 seconds). Figure 8 (a) reveals that no LPG leakage as no alarm packets are reported. Figures 8 (b) indicates 3 LPG leakage instances (the first leakage starts in the 1 st and ends in the 2 nd minute, the second leakage starts at the 3rd minutes and ends at the 4th minute, and the third leakage starts at the 6 th minute and last until the 7 th minute). The above figures reveal that our proposed system provides a robust, accurate and reliable early-detection system. Figures 9 (a) and (b) investigate the packet-loss rate as a function of packet-size and number of nodes per cluster, respectively. In terms of communication reliability, our experimental results in Figure 9 indicate that reliable and robust data delivery between the nodes with packet-loss as low as $5 \%$ is achieved.
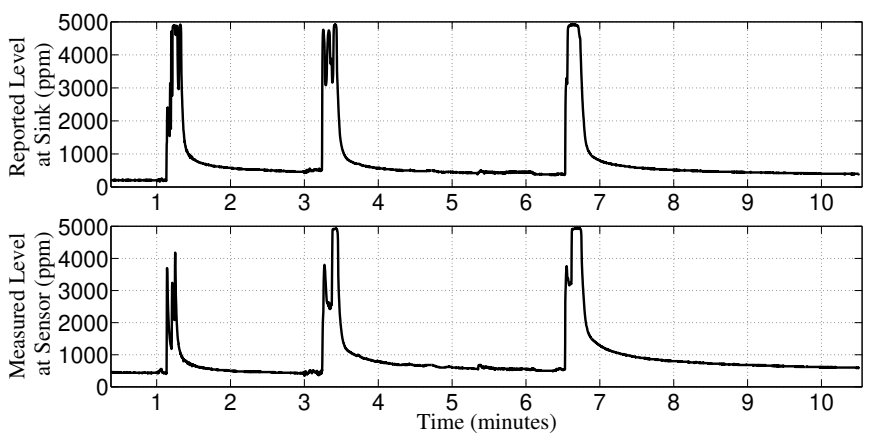

Fig. 7. The measured (at the sensor location) and reported (at the sink node) gas Level.

\section{CONCLUSIONS}

While a recently published survey paper confirms that existing LPG-detection systems are ad-hoc and designed as stand-alone nodes, this paper, presents an integrated end-toend Wireless Sensor Network (WSN) system that integrates hardware and software for early-warning gas-leakage detection and monitoring applications; fully utilizing the IoT functionalities and capabilities in WSNs at the network level such that network performance is improved. This paper presented the
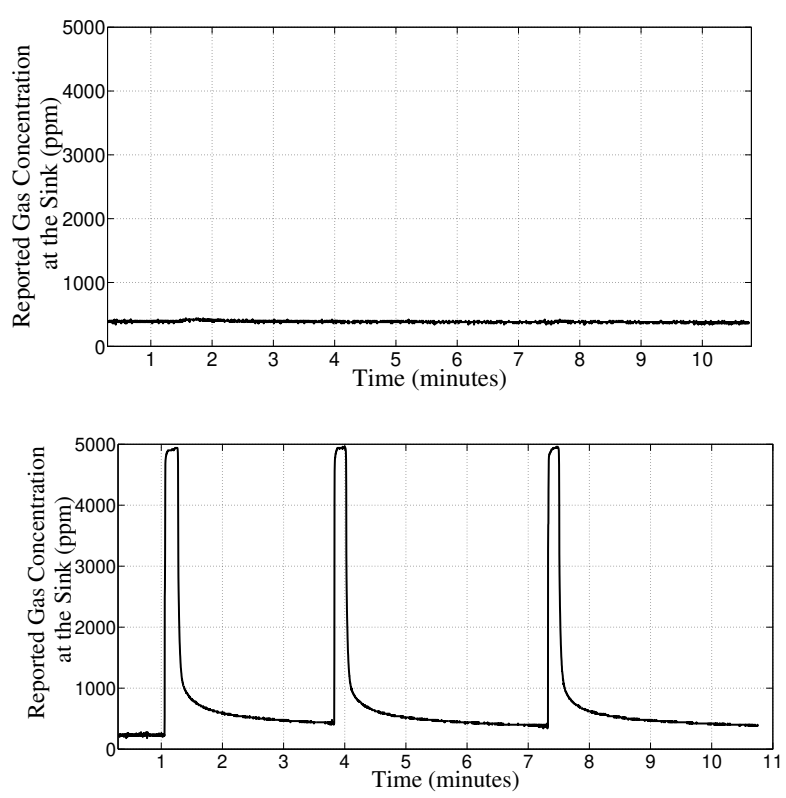

(b) Several Event

Fig. 8. Reported Gas-level at the Sink.

hardware and software components of the designed system, discussed the design of the circuit and PCB layouts for the various sensor nodes in the system, demonstrated the operation of the various developed software and communication protocols and algorithms by experimental testing. We have manufactured and assembled the various nodes in the endto-end WSN system and evaluate their performance under realistic scenarios. Specifically, the WSN prototypes are experimentally tested in order to demonstrate the effectiveness of the proposed hardware and software design components in realistic environment. The results showed that the proposed end-to-end WSN system can provide reliable, timely and efficient earlywarning event-detection system. Robust and reliable communication was demonstrated, in which the proposed end-toend system depicted a packet loss rate as low as 5\%. It is worth mentioning that the proposed system can be used in other applications to monitor and report different physical conditions (e.g., temperature, humidity and intrusion detection) by replacing the gas sensor with the appropriate sensing unit that can measure the physical conditions of interest.

\section{REFERENCES}

[1] X. Chen, S. Tang, Z. Lu, J. Wu, Y. Duan, S. Huang, Q. Tang, "iDiSC: A New Approach to IoT-Data-Intensive Service Components Deployment in Edge-Cloud-Hybrid System", IEEE Access vol. 7, pp. 59172-59184, 2019.

[2] H. Bany Salameh and R. El-Khatib, "Spectrum-Aware Routing in FullDuplex Cognitive Radio Networks: An Optimization Framework," in IEEE Systems Journal, vol. 13, no. 1, pp. 183-191, 2019. 


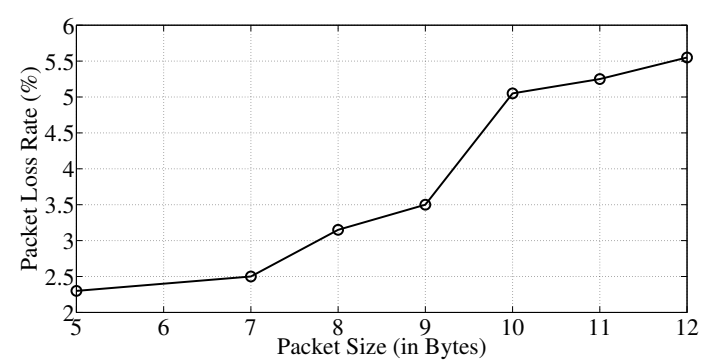

(a) Packet loss rate vs. the packet size

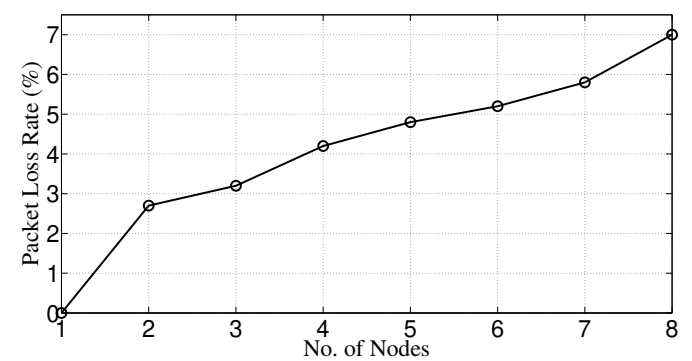

(b) Packet loss rate vs. the number of sensors per cluster

Fig. 9. Packet loss rate Performance.

[3] A. Almalaq, J. Hao, J. J. Zhang and F. Wang, "Parallel building: a complex system approach for smart building energy management," in IEEE/CAA Journal of Automatica Sinica, vol. 6, no. 6, pp. 1452-1461, 2019.

[4] H. Bany Salameh, M. AL-Quraan, "Securing Delay-sensitive CRIoT Networking under Jamming Attacks: Parallel Transmission and Batching Perspective," IEEE Internet of Things Journal, Vol. 7, iss. 8, 2020.

[5] A. Kumar, K. Ovsthus, and L. Kristensen, "An Industrial Perspective on Wireless Sensor Networks- A Survey of Requirements, Protocols, and Challenges," IEEE Communications Surveys \& Tutorials, vol. 16, no.3, pp. 1391-1412, 2014.

[6] N. Naji, M. Riduan Abid, N. Krami, D. Benhaddou, "An Energy-Aware Wireless Sensor Network for Data Acquisition in Smart Energy Efficient Building”, IEEE 5th World Forum on Internet of Things (WF-IoT), pp. 7-12, 2019.

[7] D. Deif and Y. Gadallah, "Reliable wireless sensor networks topology control for critical internet of things applications," 2018 IEEE Wireless Communications and Networking Conference (WCNC), Barcelona, 2018, pp. 1-6.

[8] X. Zhu, J. Li, and M. Zhou, "Target coverage-oriented deployment of rechargeable directional sensor networks with a mobile charger," IEEE Internet of Things Journal, Vol. 6, No. 3, pp. 5196-5208, 2019.

[9] H. Bany Salameh, T. Shu, and M. Krunz, "Adaptive cross-layer MAC design for improved energy-efficiency in multi-channel wireless sensor networks," Ad Hoc Networks Journal, Vol. 5, Iss. 6, pp. 844-854, 2007.

[10] Y. Duan, W. F. Li, X. W. Fu, Y. Luo, and L. Yang, "A methodology for reliability of WSN based on software defined network in adaptive industrial environment," IEEE/CAA J. of Autom. Sinica, vol. 5, no. 1, pp. 74-82, Jan. 2018.

[11] X. Song and Y. Li, "Data gathering in wireless sensor networks via regular low density parity check matrix," IEEE/CAA J. of Autom. Sinica, vol.5, no.1, pp. 83-91, Jan. 2018.

[12] S.E Jero, A.B. Ganesh, "PIC18LF4620 based customizable wireless sensor node to detect hazardous gas pipeline leakage," in Proc. of the International Conference on Emerging Trends in Electrical and Computer Technology, pp.563-566, 2011.

[13] A. Somov, D. Spirjakin, A. Spirjakin, A. Baranov, V. Sleptsov, and R. Passerone, "Safe delivery of sensed data in wireless sensor networks for gas leak detection: a boiler facility scenario", in Proc. of the 26th European Conference on Solid-State Transducers, 2012.

[14] H. Bany Salameh, et. al., "A Two-Level Cluster-Based Cognitive Radio Sensor Network: System Architecture, Hardware Design, and Distributed Protocols," IEEE International Conference on Cloud Engineering, AZ, 2015, pp. 287-292.

[15] M. Rakesh, S. Dagadi,"Implementation of wireless gas leakage detection system", in Proc. of the Sixth International Conference on Sensing Technology, 2012.

[16] Y. Zhang, H. Guo, Z. Lu, L. Zhan, P. C. K. Hung, "Distributed gas concentration prediction with intelligent edge devices in coal mine", Engineering Applications of Artificial Intelligence, vol. 92, pp. 103643, 2020.

[17] A. May, V. Mitchell, J. Piper, "A user centred design evaluation of the potential benefits of advanced wireless sensor networks for fire-intunnel emergency response," Fire Safety Journal, Vol. 63, pp. 79-88, 2014.

[18] A. Chunlei and T.-G. Andreas, "Applying Wireless Sensor Networks in Fire Fighting" Lecture Notes of the Institute for Computer Sciences, Social Informatics and Telecommunications Engineering, vol. 97, pp 386-395, 2012.

[19] S. Shue, J.M. Conrad, "A survey of robotic applications in wireless sensor networks," Proceedings of IEEE Southeastcon, pp.1-5, April 2013.

[20] "A WSN-Based Tool for Urban and Industrial Fire-Fighting", Sensors, 12, pp. 15009-15035, 2012.

[21] R. Suganya , P. Suseendhar, "Online Monitoring of Green House Gas Leakage in Industries", International Journal of Research in Engineering \& Advanced Technology, Vol. 1, Iss. 6, 2014.

[22] P. Jain, R. Kushwaha, "Wireless gas sensor network for detection and monitoring of harmful gases in utility areas and industries," in Proc. of the Sixth International Conference on Sensing Technology (ICST), pp.642-646, Dec. 2012.

[23] Z.A Khan, E. Pignaton de Freitas, T. Larsson, H. Abbas, "A Multiagent Model for Fire Detection in Coal Mines Using Wireless Sensor Networks," in Proc. of the 12th IEEE International Conference on Trust, Security and Privacy in Computing and Communications (TrustCom), pp.1754-1761, July 2013.

[24] H. Bany Salameh, M. Dhainat, and E. Benkhelifa, "A Survey ON Wireless Sensor Networks-based IoT Designs for Gas Leakage Detetion and Fire-fighting Applications," Jordanian Journal of Computers and Information Technology (JJCIT), vol. 5, no. 02, 2019.

[25] J. Yan, M. Zhou and Z. Ding, "Recent Advances in Energy-Efficient Routing Protocols for Wireless Sensor Networks: A Review," IEEE Access, vol. 4, pp. 5673 - 5686, Oct. 2016.

[26] H. Bany Salameh and M. Dhainat, "Event-driven Hybrid MAC Protocol for a Two-tier Cognitive Wireless Sensor Network: Design and Implementation," Int. J. of High Performance Computing and Networking, Vol. 9, Iss. 4, 2016.

[27] K. Darabkh, M. Judeh, et. al., "Mobility aware and dual phase AODV protocol with adaptive hello messages over vehicular ad hoc networks," AEU-International Journal of Electronics and Communications, vol. 94, pp. 277-292, 2018.

[28] A True System-on-chip solution for $2.4 \mathrm{GHz}$ IEEE 802.15.4/ZigBee, Texas Instruments, 2006.

[29] S. Farahani, ZigBee Wireless Networks and Transceivers, Newnespress, 2008. 
[30] IEEE Standards 802.15.4, The Institute of Electrical and Electronics Engineers, Inc. 2003.

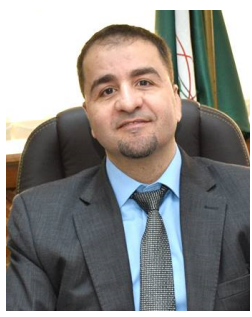

Haythem Ahmad Bany Salameh (M'06-SM'16) received the $\mathrm{Ph} . \mathrm{D}$. degree in electrical and computer engineering from the University of Arizona, USA, in 2009. He is currently a Professor of communications and networking engineering at Al Ain University, Al Ain, UAE (sabbatical leave from Yarmouk University (YU), Jordan). He also holds a visiting professor position at Staffordshire University, UK. He was a recipient of the Jordan Science Research Support Foundation (SRSF) Prestigious Award for Distinguished Research in ICT in 2015, the Best Researcher Award for Scientific Colleges in YU in 2016, and the SRSF Award for Creativity and Technological Scientific Innovation in 2017. His research interests include wireless networking, with emphasis on dynamic spectrum access, cognitive radio networking, Internet-of-Things, security, and distributed protocol design. Bany Salameh delivered several keynote lectures at different. He has served and continues to serve on the Technical Program Committee of many international conferences. prestigious venues.

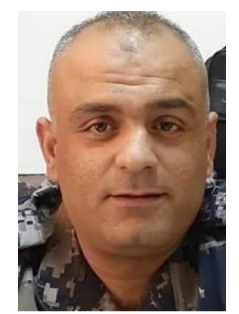

Mohammad Fozi Dhainat received the M.A. degree in wireless communications engineering from Yarmouk University, Irbid, Jordan, in 2015. He is currently working as a Senior Engineer at Civil Defence Directorate, Amman, Jordan. His research interests include cognitive radio networks, Internet of Things, and Wireless Sensor Networks.

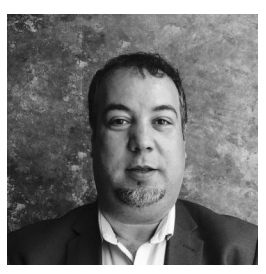

Elhadj Benkhelifa is a Full Professor of Computer Science at Staffordshire University, UK, with an extensive experience in working with industry on real world business problems. Elhadj was (20142016) the Faculty Director of the Mobile Fusion Applied Research Centre (45 PhD students and 15+ Staff). Over the past years, Elhadj has built a rich portfolio of successful collaborative cutting edge research projects, and he is currently the University Lead for REF (Research Exercise Framework) UoA11 (Computing \& Informatics). Elhadj is the Founding Head of the Cloud Computing and Applications Research Lab and the Cybersecurity Research Lab. Elhadj was a co-founding chair of several pioneering conferences/workshops. Elhadj research is very contemporary to cover many aspects of Cloud Computing including security, Mobile Cloud, Software Defined Systems, Cloud Forensics, IOT and Cloud, Fog and Mobile Edge Computing, Cloud computing resilience, Social Network Analysis, Collaborative software development, security as a service, testing as a service to mention but the most recent work. He has served as a guest Editor of many journals Special Issues, IEEE Trans Cloud Computing, Cluster Computing, Future Generation Computer System etc., and chaired several international conferences and workshops. Elhadj delivered several keynote lectures at different prestigious venues. Elhadj is a Director at Yotta Laborattories Ltd and he is a Senior R\&D Advisor to several companies in the UK and a member of several panels and committees within the UK and internationally. 